\title{
Next-to-leading order Higgs +2 jet production via gluon fusion
}

\author{
John M. Campbell, \\ Department of Physics and Astronomy, \\ University of Glasgow, Glasgow G12 8QQ, UK \\ Email: j.campbell@physics.gla.ac.uk \\ R. Keith Ellis, \\ Fermilab, Batavia, IL 60510, USA \\ and \\ PH Department, TH, CERN, 1211 Geneva 23, Switzerland \\ Email: ellis@fnal.gov \\ Giulia Zanderighi \\ PH Department, TH, CERN, 1211 Geneva 23, Switzerland \\ Email: Giulia.Zanderighi@cern.ch
}

\begin{abstract}
We present phenomenological results for the production of a Higgs boson in association with two jets at the LHC. The calculation is performed in the limit of large top mass and is accurate to next-to-leading order in the strong coupling, i.e. $\mathcal{O}\left(\alpha_{s}^{6}\right)$.

KEYWORds: Higgs, QCD, Jets.
\end{abstract}




\section{Contents}

1. Introduction 1

2. Structure of the calculation 2

3. Results 3

3.1 Inclusive cuts 3

3.2 Weak boson fusion cuts 6

4. Conclusions 10

\section{Introduction}

The search for the origin of electroweak symmetry breaking is the primary goal of the LHC programme. This breaking can be realized by the introduction of a Higgs boson whose expected physics signals and properties have been studied extensively (for example, see Ref. [1]). In particular various search strategies have been devised in order to ensure that a Higgs boson can be discovered over as large a range of putative masses as possible.

One of the main production mechanisms for the Higgs boson is the gluon fusion process shown in Figure 1(a). In this diagram we have depicted an effective coupling of the Higgs boson to gluons, which represents a top quark loop in the limit of infinite top quark mass. The Lagrangian for the effective theory is,

$$
\mathcal{L}_{\mathrm{eff}}=\frac{1}{4} A(1+\Delta) H G_{\mu \nu}^{a} G^{a \mu \nu},
$$

where $G_{\mu \nu}^{a}$ is the field strength of the gluon field, $H$ is the Higgs boson field and the effective coupling $A$ is given by

$$
A=\frac{g^{2}}{12 \pi^{2} v} .
$$

The effective coupling is thus dependent on the bare strong coupling $g$ and the vacuum expectation value parameter $v$, with $v^{2}=\left(G_{F} \sqrt{2}\right)^{-1}=(246 \mathrm{GeV})^{2}$. The finite $\mathcal{O}\left(g^{2}\right)$ correction to the effective operator has also been calculated [2, 3]

$$
\Delta=\frac{11 g^{2}}{16 \pi^{2}}+\mathcal{O}\left(g^{4}\right) .
$$

The total cross section for the production of a Higgs boson via gluon fusion at the LHC is of great importance. Consequently not only have the next-to-leading order (NLO) QCD corrections been calculated [2], but effects from one order beyond that have also been computed [ [, 5, 6, 7]. In addition the process in which one additional hard parton 


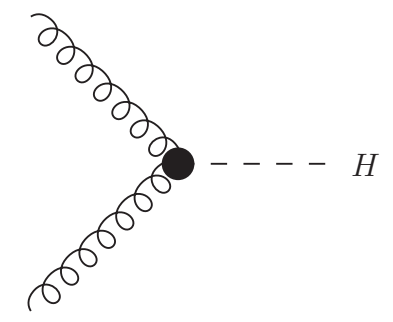

(a)

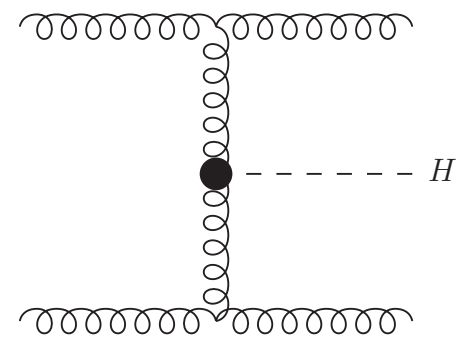

(c)

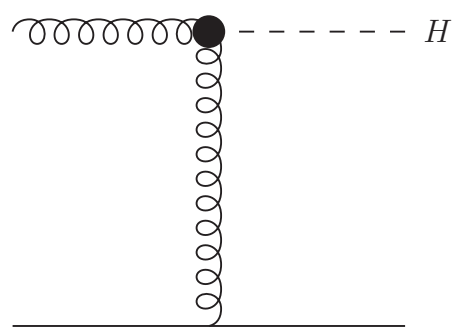

(b)

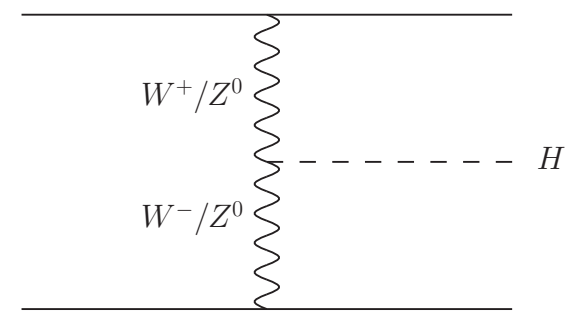

$(d)$

Figure 1: Sample diagrams representing the production of a Higgs boson at the LHC. The basic gluon fusion process is represented in diagram (a), with an additional one and two hard partons in (b) and (c) respectively. Higgs production via weak boson fusion is depicted in diagram (d).

is observed (as in Figure 1 $1(\mathrm{~b})$ ), so that the Higgs boson acquires a non-zero transverse momentum at leading order (LO), has also been calculated to NLO [8, 9, 10, 11].

It is then natural to consider the extension of this to the case in which a Higgs boson is produced in association with two hard partons [12]. There are in fact two types of processes that may contribute to such a final state, the gluon-fusion process in Figure 1(c) and the weak-boson fusion process in Figure 1(d). The latter has been known to NLO for some time 13, 14, 15]. It is the calculation of the full NLO QCD corrections to the Higgs +2 hard jets process via gluon fusion in the large $m_{t}$ approximation that we will present in this paper.

The lowest order amplitudes for Higgs +2 jet scattering have in fact been calculated exactly, without using this effective coupling [16]. This is itself a 1-loop computation involving pentagon diagrams, so the calculation is complicated considerably (and is currently intractable beyond this order). However, it allows an examination of the limits in which the effective coupling approach is valid. The results of this study indicate that this approach is accurate as long as $m_{H}, p_{T}$ (jet) $<m_{t}$, a condition which will be satisfied for the results presented in this paper.

\section{Structure of the calculation}

A detailed description of the calculation of the virtual matrix elements using a seminumerical approach is given in Refs. [17, 18]. 
The real matrix element corrections to $H+2$ jet production are obtained by including all crossings of results for the three basic processes,

$$
\begin{aligned}
& \text { A) } 0 \rightarrow H q \bar{q} q^{\prime} \bar{q}^{\prime} g, \\
& \text { B) } 0 \rightarrow H q \bar{q} g g g \\
& \text { C) } 0 \rightarrow H g g g g g .
\end{aligned}
$$

For process $A$ ) the relevant matrix elements of Ref. [19] are implemented. We have also used the results therein for process $B$ ) when the helicities of all gluons are the same. For all other helicity assignments we have chosen to use the more compact representation provided by the MHV techniques of Ref. [20]. Finally, process $C$ ) exploits the NNMHV matrix elements of Ref. [21], but uses the results of Del Duca et al. for the other helicity combinations. Soft and collinear singularities are handled using the dipole subtraction scheme [22].

The calculation is incorporated into the general-purpose next-to-leading order code MCFM.

\section{Results}

In order to render the cross section finite, we must apply some simple cuts to the jets. The choice of cuts that we make is motivated partly by the studies of Ref. [15], in which the sensitivity of the Higgs cross section via the QCD and weak boson fusion processes to the choice of minimum jet transverse momentum is studied. For this choice of cuts, the cross section for the production of a Higgs boson and two or more jets is dominated by the $H+2$ jet contribution. As a result, the NLO QCD cross section for this process shows the usual reduced dependence on the renormalization and factorization scales.

\subsection{Inclusive cuts}

The simplest "inclusive" set of cuts that we consider is specified by the following constraints on the jets, which are formed from the partons according to the usual $k_{T}$-clustering algorithm [23]:

$$
p_{t}(\text { jet })>40 \mathrm{GeV}, \quad\left|\eta_{\text {jet }}\right|<4.5, \quad R_{\text {jet,jet }}>0.8 .
$$

All of our results are based upon events in which at least two jets satisfy these cuts, with the additional parton appearing at NLO sometimes manifest as a third jet. We do not consider the decay of the Higgs boson and apply no cuts directly to the Higgs boson itself.

Before presenting any results, we note that the choice of parton distribution function (PDF) that is used in the calculation is crucial. Each PDF set is obtained by fitting a collection of observables with a particular value of $\alpha_{s}\left(m_{Z}\right)$. Since the effective coupling of the Higgs field to two gluons is of order $\alpha_{s}$ (Eq. 1.2), the final lowest order matrix elements

squared for the $H+2$ jet process are proportional to $\alpha_{s}^{4}$ and thus the cross section is very sensitive to the input value from the PDF set. Throughout this paper we will use two sets from the CTEQ6 package [24]. At leading order (LO) the CTEQ6L1 set is used (with 
$\alpha_{s}\left(m_{Z}\right)=0.130$ and 1-loop running) whilst at NLO we have performed the calculations with CTEQ6M $\left(\alpha_{s}\left(m_{Z}\right)=0.118,2\right.$-loop running).

Higgs production via the weak boson fusion (WBF) process is most interesting for "intermediate" masses, in the region $115<m_{H}<160 \mathrm{GeV}$. Since the QCD production mechanism that we discuss in this paper is mostly of interest as a source of additional events containing Higgs bosons in such a WBF analysis, we limit our study to the same range. We choose the Higgs masses at either end of the range and note that the effective $H g g$ coupling that we have used remains a good approximation for these values of $m_{H}{ }^{1}$.

Our results for the cross sections with the cuts given by Eq. (3.1) are summarized in Table 1. Cross sections for the weak boson fusion process at NLO are also shown in Table 1. They are about half the size of those for the gluon fusion process. The default value for both the renormalization and factorization scales is $m_{H}$. With this choice of scale and the cuts of Eq. 3.1, the NLO corrections to the $H+2$ jet cross section are quite mild, increasing the LO cross section by only $15 \%$ for the lower

\begin{tabular}{lll} 
Higgs mass & $115 \mathrm{GeV}$ & $160 \mathrm{GeV}$ \\
\hline$\sigma_{\mathrm{LO}}[\mathrm{pb}]$ & 3.50 & 2.19 \\
$\sigma_{\mathrm{NLO}}[\mathrm{pb}]$ & 4.03 & 2.76 \\
\hline$\sigma_{\mathrm{WBF}}[\mathrm{pb}]$ & 1.77 & 1.32
\end{tabular}

Table 1: LO and NLO cross sections for the gluon-fusion process with the basic inclusive cuts of (Eq. 3.1), together with the weak boson fusion cross section at NLO.

mass and by $26 \%$ for a $160 \mathrm{GeV}$ Higgs boson. Although the corrections to the $H+2$ jet cross section via gluon fusion are a little larger than those for the corresponding WBF process (which are around 10\%), existing LO analyses would not be much changed by the inclusion of NLO corrections via such an inclusive $K$-factor. In contrast, the $H+1$ jet cross section, using $m_{H}=115 \mathrm{GeV}$ and the same choice of jet definition, increases by about $50 \%$ at NLO.

The dependence of the cross section on the unphysical renormalization and factorization scales can be used to assess not only the sensitivity of physical predictions to these inputs, but also the trustworthiness of the perturbative series itself. To that end, we show in Figure 2 the scale dependence obtained from our LO and NLO predictions, for each choice of Higgs mass. The dependence on each scale is shown separately, with a variation by a factor of two about the default value of $m_{H}$, while the other scale is held fixed. The shapes of the curves for the two Higgs masses are very similar. In each case the dependence of both the LO and NLO cross sections on the factorization scale is very mild, with a slightly smaller variation at NLO. In contrast, the dependence on the renormalization scale is very significant at LO, as expected. This is somewhat reduced at NLO, although a large dependence remains, with the cross section increasing by about $35 \%$ when the renormalization scale is halved. Therefore the NLO cross section still contains a significant residual uncertainty that should be accounted for in an honest analysis. This intrinsic lack of reliability is in stark contrast to the WBF process, which contains very little dependence on the input scales. This is demonstrated in Figure 3, where we show the behaviour of the NLO cross section for production of a Higgs boson by WBF, under the same set of cuts.

\footnotetext{
${ }^{1}$ It is possible to approximate the effects of performing the full calculation at finite $m_{t}$ by scaling all our results by the ratio of the leading order cross sections in each approach, according to the results of Ref. [16]. Since the difference is at most only a few percent for $m_{H}=160 \mathrm{GeV}$, we have not done so here.
} 

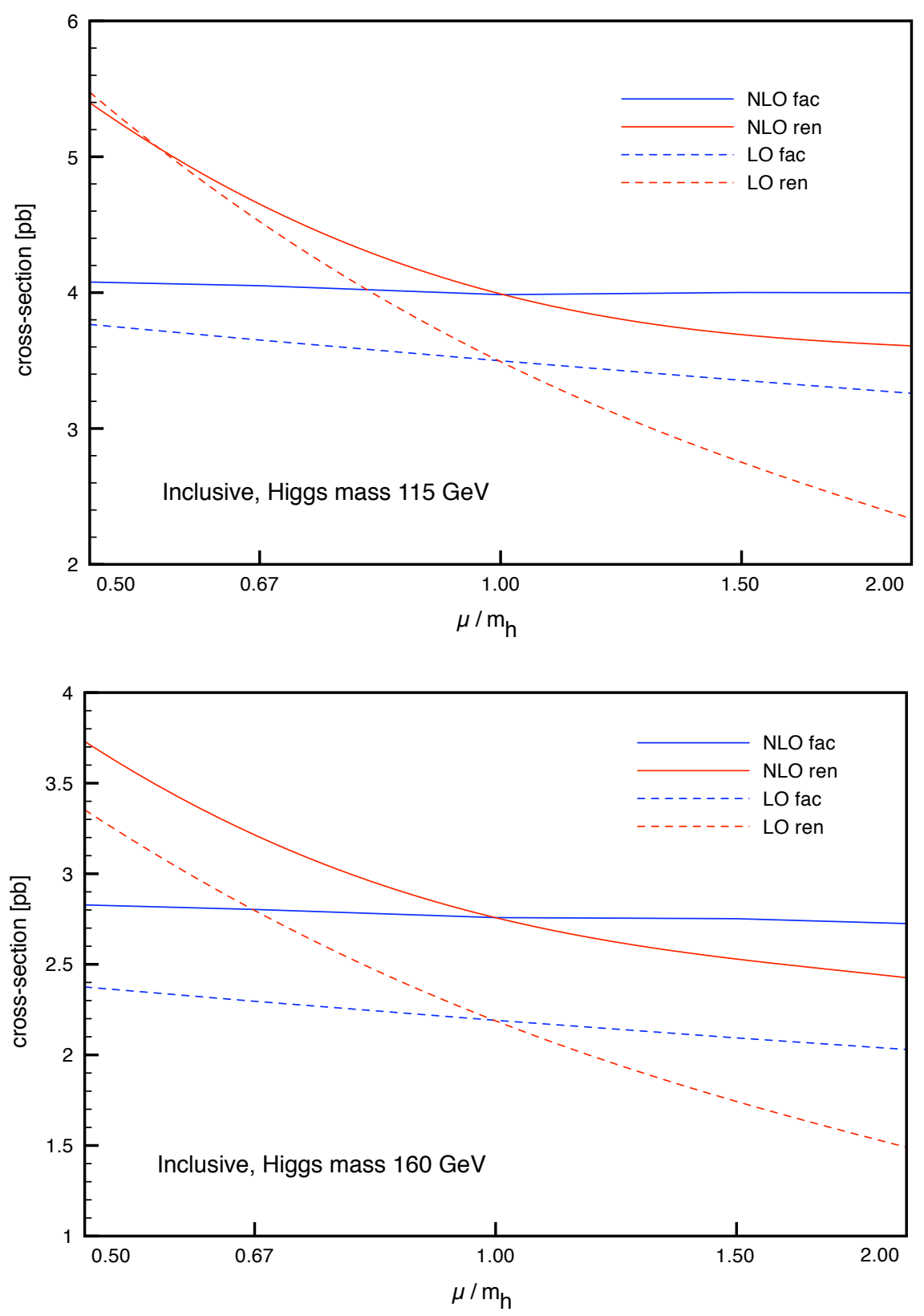

Figure 2: Scale dependence of the Higgs +2 jet cross section with minimal rapidity and transverse momentum cuts, for $m_{H}=115 \mathrm{GeV}$ (upper) and $m_{H}=160 \mathrm{GeV}$ (lower).

For both masses, the cross section varies only by approximately $1.5 \%$ over the same range of scales as considered above.

We note that a choice for the minimum jet transverse momentum lower than given in Eq. (3.1) results in similar scale-dependence plots, but with much steeper renormalization scale curves. We interpret this as a sign that the perturbative series is less well-behaved for such a choice of cuts. Indeed, in those cases, the cross section for producing a Higgs +3 jet 


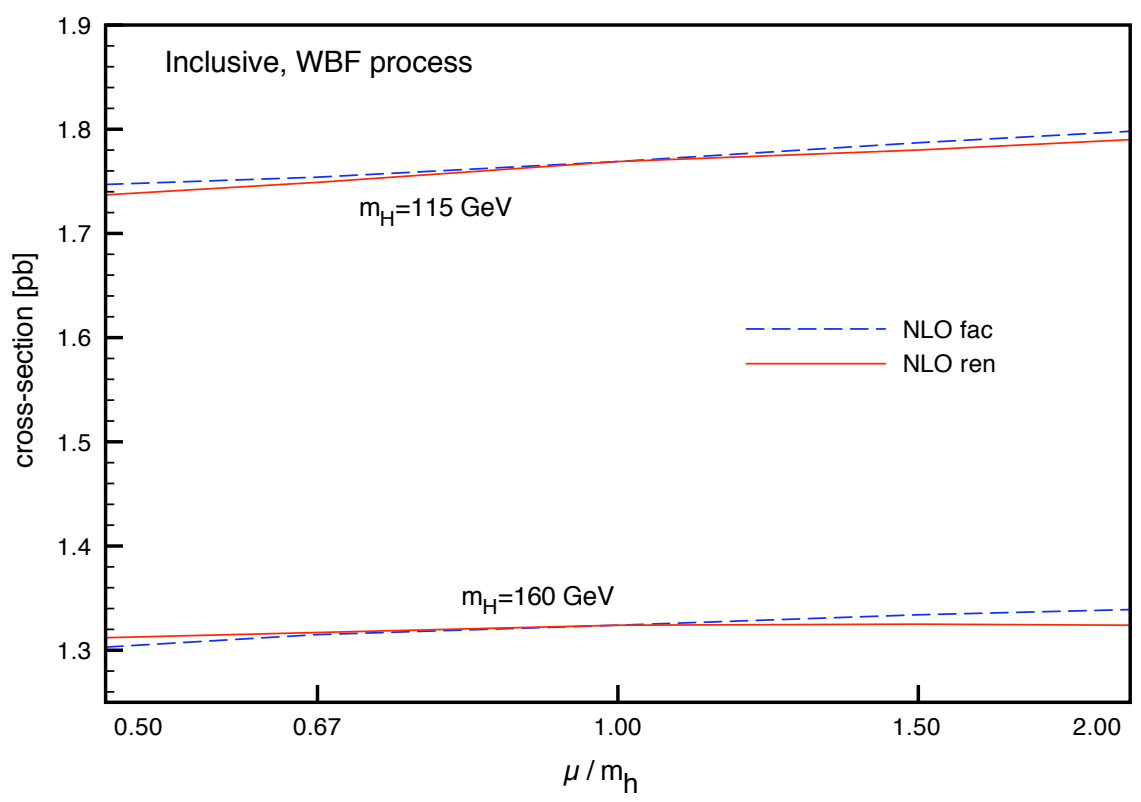

Figure 3: Scale dependence of the weak boson fusion Higgs cross section with minimal rapidity and transverse momentum cuts, for $m_{H}=115 \mathrm{GeV}$ (upper set of curves) and $m_{H}=160 \mathrm{GeV}$ (lower).

final state becomes larger than the one for Higgs +2 jets.

The calculation that we have performed can be used to study much more than the twojet inclusive cross section above. In the analysis of Higgs production via weak boson fusion, it is imperative to study the distribution of the rapidities of the jets that are produced and in particular, whether or not this is changed by NLO corrections. It is clearly prudent to perform the same analysis for the process at hand. In Figure 4 we present the results of such a study. In this study the two jets in each event with the largest transverse momenta are chosen. Their rapidities are entered into the histogram with weight one half, so that the area under the histograms yield the NLO cross sections shown in Table 1. The LO distribution has been scaled up by the ratio of cross sections in that table, so that its area is the same. The distribution shows no evidence for any change of shape when including the QCD corrections. The figure also shows the NLO prediction for the weak boson fusion process, which is significantly different. Even with this very minimal set of cuts, it is clear that requiring just one of the two jets to be produced at a relatively large rapidity (for example, 2 units) significantly enhances the production of Higgs bosons via WBF with respect to the QCD production mechanism.

\subsection{Weak boson fusion cuts}

In addition to the basic cuts discussed above, we have also considered a set of cuts that is designed to enhance the weak boson fusion Higgs process and suppress processes which involve the production of additional jets via QCD. There are many variants of these cuts, all designed to pick out configurations involving one or more forward jets. We choose a 

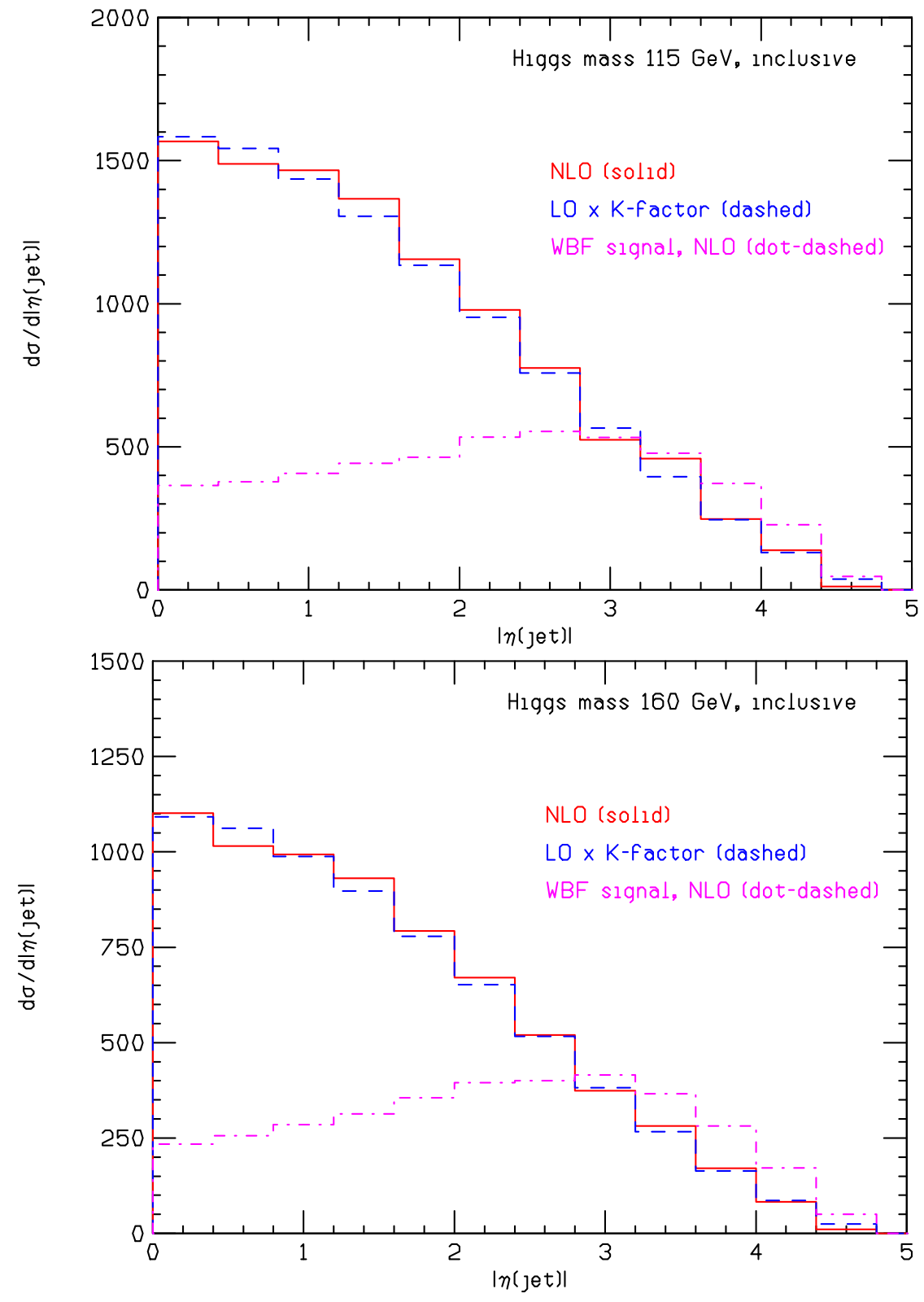

Figure 4: The pseudorapidity distribution of the two leading jets using only the inclusive cuts, for $m_{H}=115 \mathrm{GeV}$ (upper) and $m_{H}=160 \mathrm{GeV}$ (lower). The QCD process is calculated at LO (rescaled by the inclusive $K$-factor) and at NLO. The NLO result for weak boson fusion is also shown for comparison. Both jets in an event enter the histogram, each with weight one half.

fairly minimal set of constraints on the two jets with the highest transverse momenta $\left(j_{1}\right.$ and $j_{2}$ ), in addition to the cuts in Eq. 3.1, we impose

$$
\left|\eta_{j_{1}}-\eta_{j_{2}}\right|>4.2, \quad \eta_{j_{1}} \cdot \eta_{j_{2}}<0
$$

Thus the two "tagging jets" are required to be both well-separated in rapidity and to lie in opposite hemispheres. Note that we have refrained from using the term "rapidity gap" 
to describe this separation, since the additional softer parton that can be present at NLO may lie between the two tagging jets.

The cross sections that we find when using this set of cuts are shown in Table 2. We again choose both renormalization and factorization scales equal to $m_{H}$ for these predictions. From the lowest order cross sections it is clear that this set of cuts probes a rather small fraction, less than $8 \%$, of the total cross section in Table 1. For this reason it is somewhat harder to perform the integration over the phase space at NLO and we have thus indicated the statistical errors from our numerical integra-

\begin{tabular}{lll} 
Higgs mass & $115 \mathrm{GeV}$ & $160 \mathrm{GeV}$ \\
\hline$\sigma_{\mathrm{LO}}[\mathrm{fb}]$ & 271 & 172 \\
$\sigma_{\mathrm{NLO}}[\mathrm{fb}]$ & $346 \pm 5$ & $236 \pm 3$ \\
\hline$\sigma_{\mathrm{WBF}}[\mathrm{fb}]$ & 911 & 731
\end{tabular}

Table 2: LO and NLO cross sections with the weak boson fusion search cuts of Eq. (3.2). Errors on the NLO cross sections are statistical only. Also shown are the WBF cross sections at NLO. tion in the table. These errors are at the level of $1.5 \%$. With these cuts, the effect of the QCD corrections is greater than in the more inclusive case. The cross section increases by about $30 \%$ for $m_{H}=115 \mathrm{GeV}$ and by a little more, around $40 \%$, for the higher mass. In this table we also show the corresponding cross sections for the weak boson fusion process, for comparison. One can see that the cuts of Eq. (3.2) have been quite effective in suppressing the gluon-fusion process, so that the cross sections due to weak boson fusion dominate by about a factor of 2.5 .

We also repeat the scale dependence study that was performed with the inclusive cuts in the analogous Figure 5 . The results are broadly similar, with the cross section showing little factorization scale dependence but a considerable variation with the renormalization scale. Again this renormalization scale dependence is decreased somewhat at NLO, but a large uncertainty on the cross section remains.

Of particular interest to the Higgs boson search is the distribution of the azimuthal angle between the two accompanying jets $(\Delta \phi)$. Because of the CP-even (scalar) nature of the Higgs boson, the QCD process which we consider in this paper produces a large correlation between these two jets, with a $\Delta \phi$ distribution that is peaked at 0 and $\pi$ and heavily suppressed at $\Delta \phi=\pi / 2$. This is in contrast to the weak boson fusion process, which produces an almost flat distribution in $\Delta \phi$. Therefore this observable has been considered as an additional discriminator between the two processes. Moreover, a CPodd (pseudoscalar) Higgs boson would produce a very different distribution in the QCD process (with the position of the peaks and trough reversed), so that this mechanism could potentially be used to probe the $\mathrm{CP}$ properties of the Higgs.

These observations pertain to the lowest order predictions. It is natural to consider whether or not they still apply in more detailed studies. An investigation of the effects of a parton shower [25] suggested that $\Delta \phi$ is subject to logarithmically-enhanced higher order corrections and the correlation is reduced. A more recent study, aiming to separate the effects of hard radiation from those of showering and hadronisation [26], finds that the correlation largely survives both effects. With the calculation presented in this paper we are able to provide further insight by studying the effects of the NLO corrections to this observable.

The results of our study are shown in Figure 6, where we have again scaled up the 

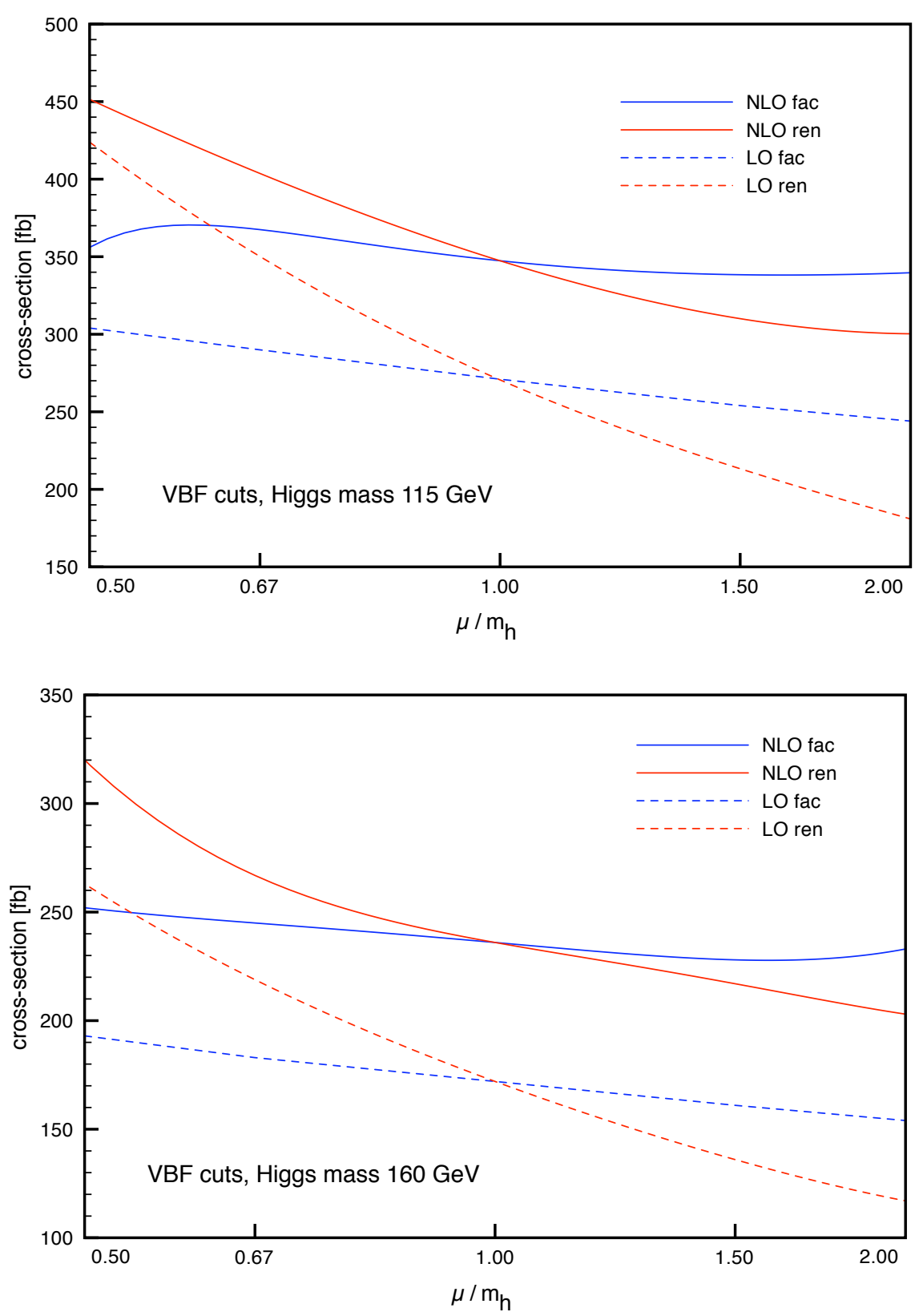

Figure 5: Scale dependence of the Higgs +2 jet cross section in the region selected by the weak boson fusion cuts, for $m_{H}=115 \mathrm{GeV}$ (upper) and $m_{H}=160 \mathrm{GeV}$ (lower).

lowest order prediction by the ratio of cross sections in Table2, so as to enable a comparison of the shapes of this distribution. Our results indicate that the shape of the lowest order distribution is unchanged and therefore that the correlation survives the addition of NLO QCD corrections. 

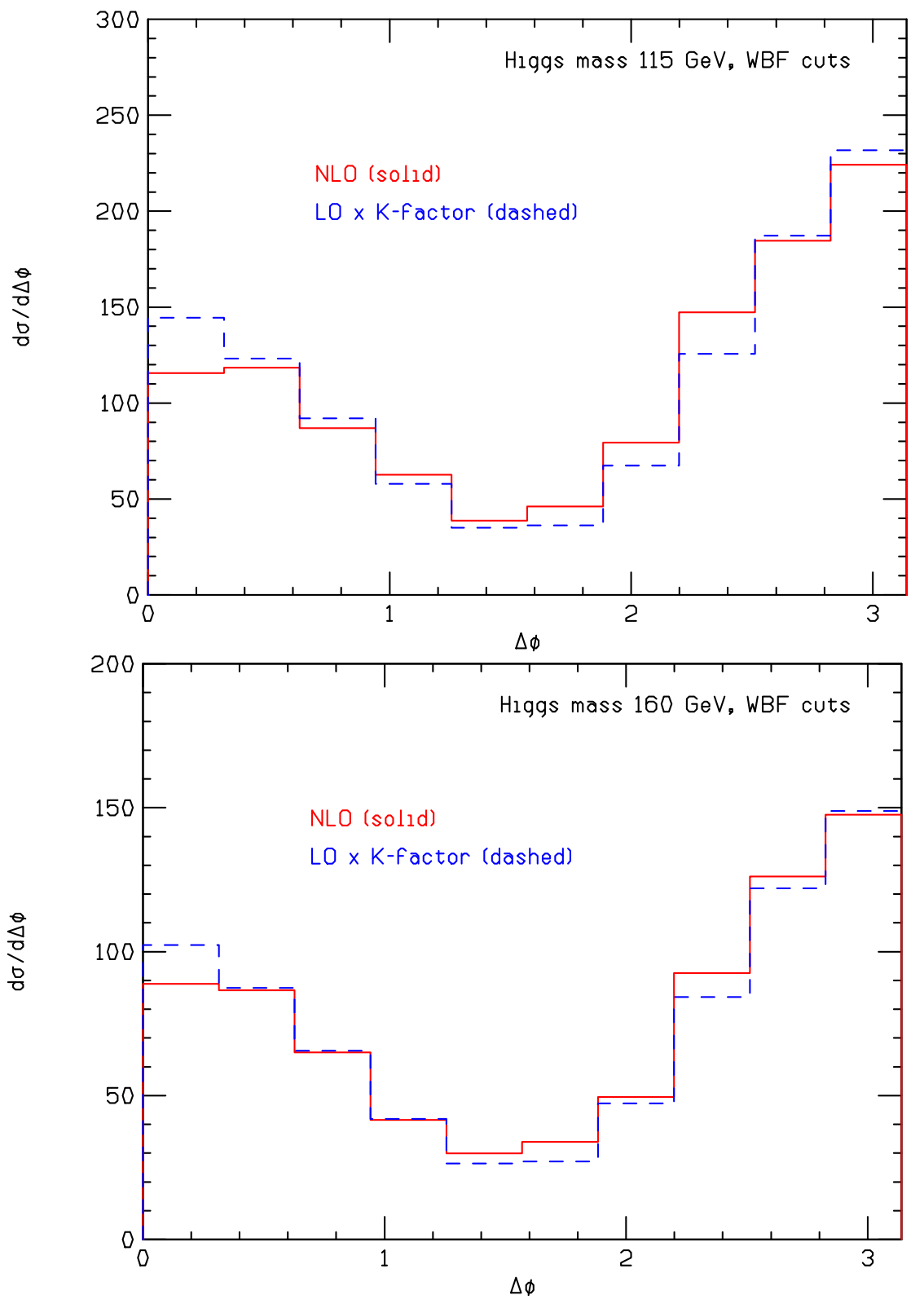

Figure 6: The azimuthal correlation of the two leading jets using the weak boson fusion cuts, for $m_{H}=115 \mathrm{GeV}$ (upper) and $m_{H}=160 \mathrm{GeV}$ (lower). The QCD process is calculated at NLO and LO, with the latter scaled up by the overall $K$-factor in this region.

\section{Conclusions}

We have presented a calculation of the production of a Higgs boson in association with two jets at hadron colliders, performed in the limit of large top mass and accurate to the next-to-leading order in the strong coupling. Our results indicate that the effect of the QCD corrections is modest and that the dependence of observables on the factorization and renormalization scales is reduced. Furthermore we find that the azimuthal correlation between the two leading jets, the subject of recent parton shower based studies, is 
unchanged at NLO.

A major source of error in the extraction of the Higgs coupling to vector bosons at the LHC is the theoretical uncertainty on the $H+2$ jet gluon fusion process. Previous studies have estimated this uncertainty to be $20 \%$ [27] or 30\% [15]. Our results suggest that the uncertainty due to the scale dependence alone may be at least as large as this. Further study is necessary in order to extract the coupling with greater confidence.

In the wider context, this calculation represents the first full implementation of the semi-numerical approach [17 for the virtual matrix elements.

\section{Acknowledgments}

We are grateful to Walter Giele for useful discussions. We would like to thank Vittorio Del Duca, Alberto Frizzo and Fabio Maltoni for providing us with their code for the evaluation of the $H+5$ parton matrix elements and Daniel De Florian for comments on the manuscript.

\section{References}

[1] A. Djouadi, arXiv:hep-ph/0503172.

[2] S. Dawson, Nucl. Phys. B 359 (1991) 283.

[3] A. Djouadi, M. Spira and P. M. Zerwas, Phys. Lett. B 264 (1991) 440.

[4] R. V. Harlander and W. B. Kilgore, Phys. Rev. Lett. 88 (2002) 201801 [arXiv:hep-ph/0201206].

[5] C. Anastasiou and K. Melnikov, Nucl. Phys. B 646 (2002) 220 [arXiv:hep-ph/0207004].

[6] V. Ravindran, J. Smith and W. L. van Neerven, 'NNLO corrections to the total cross section for Higgs boson production in Nucl. Phys. B 665 (2003) 325 [arXiv:hep-ph/0302135].

[7] C. Anastasiou, K. Melnikov and F. Petriello, Nucl. Phys. B 724 (2005) 197 [arXiv:hep-ph/0501130].

[8] C. R. Schmidt, Phys. Lett. B 413 (1997) 391 [arXiv:hep-ph/9707448].

[9] D. de Florian, M. Grazzini and Z. Kunszt, Phys. Rev. Lett. 82, 5209 (1999) [arXiv:hep-ph/9902483].

[10] V. Ravindran, J. Smith and W. L. Van Neerven, Nucl. Phys. B 634 (2002) 247 [arXiv:hep-ph/0201114].

[11] C. J. Glosser and C. R. Schmidt, JHEP 0212, 016 (2002) [arXiv:hep-ph/0209248].

[12] R. P. Kauffman, S. V. Desai and D. Risal, [Erratum-ibid. D 58 (1998) 119901] [arXiv:hep-ph/9610541].

[13] T. Han, G. Valencia and S. Willenbrock, Phys. Rev. Lett. 69 (1992) 3274 [arXiv:hep-ph/9206246].

[14] T. Figy, C. Oleari and D. Zeppenfeld, Phys. Rev. D 68 (2003) 073005 [arXiv:hep-ph/0306109].

[15] E. L. Berger and J. Campbell, Phys. Rev. D 70 (2004) 073011 [arXiv:hep-ph/0403194]. 
[16] V. Del Duca, W. Kilgore, C. Oleari, C. Schmidt and D. Zeppenfeld, Nucl. Phys. B 616 (2001) 367 [arXiv:hep-ph/0108030].

[17] R. K. Ellis, W. T. Giele and G. Zanderighi, Phys. Rev. D 73 (2006) 014027 [arXiv:hep-ph/0508308].

[18] R. K. Ellis, W. T. Giele and G. Zanderighi, Phys. Rev. D 72 (2005) 054018 [arXiv:hep-ph/0506196].

[19] V. Del Duca, A. Frizzo and F. Maltoni, JHEP 0405 (2004) 064 [arXiv:hep-ph/0404013].

[20] S. D. Badger, E. W. N. Glover and V. V. Khoze, JHEP 0503 (2005) 023 [arXiv:hep-th/0412275].

[21] L. J. Dixon, E. W. N. Glover and V. V. Khoze, JHEP 0412 (2004) 015 [arXiv:hep-th/0411092].

[22] S. Catani and M. H. Seymour, Nucl. Phys. B 485 (1997) 291 [Erratum-ibid. B 510 (1997) 291] [arXiv:hep-ph/9605323].

[23] G. C. Blazey et al., arXiv:hep-ex/0005012.

[24] J. Pumplin, D. R. Stump, J. Huston, H. L. Lai, P. Nadolsky and W. K. Tung, JHEP 0207, 012 (2002) [arXiv:hep-ph/0201195].

[25] K. Odagiri, JHEP 0303, 009 (2003) [arXiv:hep-ph/0212215].

[26] V. Del Duca et al., arXiv:hep-ph/0608158.

[27] D. Zeppenfeld, R. Kinnunen, A. Nikitenko and E. Richter-Was, Phys. Rev. D 62 (2000) 013009 [arXiv:hep-ph/0002036]. 